\title{
Chronic Pain Assessment in Moroccan Hemodialysis Population
}

\author{
Ibtihale Boukhira ${ }^{1 *}$, Said Jidane ${ }^{1}$, Ahmed Kharbach ${ }^{2}$, Lahcen Belyamani ${ }^{1}$
}

\begin{abstract}
${ }^{1}$ Research team in analgesia and local loco anesthesia, Faculty of Medicine and Pharmacy of Rabat, University Mohamed 5, Rabat, MOROCCO
${ }^{2}$ Laboratory of Biostatistics, Clinical Research and Epidemiology (LBRCE), Faculty of Medicine and Pharmacy of Rabat, Mohammed V University of Rabat, MOROCCO ${ }^{\star}$ Corresponding Author: ibtihalee@gmail.com
\end{abstract}

Citation: Boukhira I. Chronic Pain Assessment in Moroccan Hemodialysis Population. Electron J Gen Med. 2021;18(6):em325. https://doi.org/10.29333/ejgm/11312

\section{ARTICLE INFO}

Received: 5 Sep. 2021

Accepted: 19 Oct. 2021

\begin{abstract}
Introduction: This study aims to assess the prevalence, as well as the impact of chronic pain on the daily life of hemodialysis patients and to determine the associated socio-demographic, clinical-biological, and psychological factors.

Methods: This study was a cross-sectional study that included 441 hemodialysis patients. The characteristics of the chronic pain were collected by the Brief Pain Inventory questionnaire and by the visual analog scale. The anxious and depressed mood was assessed by the Hospital Anxiety and Depression Scale, factors associated with chronic pain were determined through univariate and multivariate analysis.

Results: the prevalence of chronic pain was $72.8 \%$. It was severe in $40.8 \%$ of cases and it completely interfered with general activity in $72.9 \%$ of cases. The most frequently reported pain site was: lowers extremities (39.9\%). Thus, $59.9 \%$ of patients reported using analgesics, with a frequent intake in $74.3 \%$ of cases. Anxiety and depression were found respectively in $68 \%$ and $66 \%$ of cases. The chronic pain was significantly associated with depression $(p<0.001)$, anxiety $(p<0.001)$, living conditions ( $p<0.001)$, level of studies $(p<0.001)$, and marital status $(p=0.020)$.
\end{abstract}

Conclusion: A multidisciplinary approach is recommended for the management of chronic pain, involves Nurses, physiotherapists, psychologists, health educators and family.

Keywords: chronic pain, hemodialysis, assessement, associated factors

\section{INTRODUCTION}

Chronic pain is a common, complex, and distressing problem that has a profound impact on individuals and society [1]. Indeed, it's a source of disability, major social and psychological alterations [2], and even more in hemodialysis patients.

The prevalence of chronic pain in hemodialysis patients was up to $82 \%$ and $92 \%[3,4]$, and it is severe to moderate in about $35 \%$ to $70 \%$ of these patients [4].

However, in chronically hemodialyzed population, most patients are not evaluated for these aspects and therefore not sufficiently treated [5]. Health professionals should therefore understand and relieve pain in this population, in order to improve their quality of life and care [4]. Chronic pain must be understood in the context of social, biological, psychological, and physical factors in order to develop treatment plans and prevention strategies [1]. It is important to analyze these different factors for adequate and holistic pain management.

This study aims to assess the prevalence, as well as the impact of chronic pain on the daily life of hemodialysis patients, in order to determine the associated sociodemographic, clinical-biological, and psychological factors.

\section{PATIENTS AND METHODS}

\section{Ethics Approval and Consent to Participate}

The study has been approved by the ethics committee for biomedical research of the MOHAMMED V Faculty of Medicine and Pharmacy in RABAT (N/R: Folder Number 10/20), and informed consent was obtained from each subject.

\section{Design and Study Area}

This is a multicenter cross-sectional study conducted from February 2020 to September 2020 including all hemodialysis patients $(n=441)$ recruited from all public sectors hemodialysis centers ( 6 centers) in Souss Massa region, Morocco.

\section{Inclusion and Exclusion Criteria}

Patients satisfying the following criteria were included in this study consecutively: age greater than 18 years old, Moroccan nationality, time on hemodialysis greater than three months, absence of a recent change in the usual lifestyle, and a prior agreement. They will be excluded in this study: Hemodialysis patients who are comatose, delusional and nonconsenting.

\section{Chronic Pain Definition}

Pain is defined by duration, it is considered chronic if it persists more than three months [6], From this criterion and based on the pain syndrome, we divided our population into 
two groups, according to the presence or absence of chronic pain.

\section{Instrument and Data Collection}

Basic demographic data were collected, including information about age, gender, health cover, level of education, marital status, professional one, and living conditions. Baseline clinical, hematological, dialytic, and psychological data of these patients were also collected: clinical (causal nephropathy, associated comorbidities, body mass index, toxics habits, and respect of hygieno-dietetic rules), biological (hemoglobin, phosphatemia, albumin, Creactive protein, mean calcium level, thyroid assessment), dialytics (duration of hemodialysis, number of dialysis sessions, Interdialytic Weight Gain, Vascular Access, and Renal therapy replacement), and psychological (depression and anxiety).

In this study, the characteristics of the chronic pain were collected by the BPI questionnaire: Brief Pain Inventory (Short Form), with the Arabic version which is already validated [7], (type, intensity, site, frequency, origin, psycho-affective impact on the daily life of hemodialysis patients).

Pain intensity was assessed using the visual analog scale (VAS) allowing patients to choose a number from 0 to 10 to describe the degree of their chronic pain, then it was classified as absent for a score of 0 , low for a score from 1 to 3 , moderate for a score of 4 to 6 , severe for a score of 7 to 9 and unbearable for a score of 10. Regarding the frequency, chronic pain is considered as permanent if it was present continuously without no-pain interval, daily if it occurs at least once a day, intermittent if it occurs less than once a day, and finally, rare when it occurs less than once a week.

The HADS scale (Hospital Anxiety and Depressive scale) in its already validated Arabic version [8] is used to detect depression and anxiety in our population. It is a reliable instrument to verify the presence or absence of depression and anxiety and can even measure the severity of anxiety and depressive disorders [9] . It comprises 14 items graded from 0 to 3. Seven questions relate to anxiety (total A) and seven others to the depressive dimension (total D), thus, making it possible to obtain two scores (maximum score for each score $=$ 21). The anxiety score is obtained by adding the scores assigned to the questions on anxiety. A score greater than or equal to 11 defines anxiety. The depression score is obtained by adding the scores assigned to the seven questions on depression. A score greater than or equal to 11 defines depression [9].

\section{Data Management and Statistical Analysis}

The qualitative variables were presented as frequency and percentages, and mean \pm standard deviation (SD) or median (interquartile range, IQR) for quantitative variables. The Chisquare test $\left(x_{2}\right)$ or Fisher's exact test, were performed according to their particular application conditions, to examine for differences in proportions of categorical variables between two groups (the group with chronic pain and the group that does not complain of any chronic pain). Furthermore, univariate and multivariate logistic regression analyses were done to identify the factors associated with chronic pain. All independent variables with a $P$-value $<.25$ in the univariate analysis were taken into account in the multivariate logistic regression analysis.

The difference is considered statistically significant for a $P$ $<$.05. Data management and statistical analysis was done using the SPSS for Windows software package (ver. 13.0; SPSS Inc., Chicago, IL, USA).

\section{RESULTS}

\section{Characteristics of the Study Population}

The total included in the study was 441 of chronic hemodialysis patients, with a mean age of 56.05 (15.67). Elderly subjects (> 65 years) represented $30.6 \%$ of patients $(n=135)$. The sex ratio M / F was 1.29 (249 M / 192 F), the majority (63.7 $\%)$ of the participants was illiterate. $61.2 \%$ of patients were living alone or with one person in their house and almost all of the participants $(85 \%)$ were unemployed. There were $300(68$ $\%)$ patients who self-reported anxiety, and 291 (66\%) depression. However, only $2.7 \%$ reported using anxiolytics and $2.3 \%$ antidepressants. Table 1 summarizes all the sociodemographic and psychological characteristics of patients.

The mean body mass index (BMI) was 23.18 (3.6) and the median is $24.03(21.60-25.90) \mathrm{kg} / \mathrm{m}^{2}$. The mean and median duration of hemodialysis are $64.84 \pm 49.67$ months and 56 (IQR 28-84) months, respectively. The etiologies of end-stage

Table 1. Socio-demographic and psychological characteristics of the patients classified according to the presence of chronic pain

\begin{tabular}{|c|c|c|c|c|}
\hline Variable & $\begin{array}{c}\text { All patients } \\
\mathrm{N}(\%)\end{array}$ & $\begin{array}{c}\text { Chronic pain } \\
\mathrm{N}(\%) \\
\end{array}$ & $\begin{array}{c}\text { No Chronic pain } \\
\mathrm{N}(\%)\end{array}$ & $P$ value \\
\hline Age & $56.05 \pm 15.67$ & $57.04 \pm 15.28$ & $52.40 \pm 16.16$ & $<0.01^{\star}$ \\
\hline [18-45 years] & $113(25.6)$ & 70 (15.9) & $43(9.8)$ & \\
\hline [46-65 years] & $193(43.8)$ & $144(32.7)$ & $49(11.1)$ & \\
\hline$>65$ years & $135(30.6)$ & $107(24.3)$ & $28(6.3)$ & \\
\hline Gender & & & & 0.072 \\
\hline Male & 249 (56.5) & $174(39.5)$ & $75(17)$ & \\
\hline Female & $192(43.5)$ & 147 (33.3) & $45(10.2)$ & \\
\hline Level of studies & & & & $<0.01^{\star}$ \\
\hline Illiterate & $281(63.7)$ & $206(64.2)$ & $75(62.5)$ & \\
\hline Primary & $79(17.9)$ & $61(19)$ & $18(15)$ & \\
\hline Middle school & $36(8.2)$ & $19(5.9)$ & $17(14.2)$ & \\
\hline Hight school & $41(9.3)$ & $34(10.6)$ & $7(5.8)$ & \\
\hline Higher Education & $4(0.9)$ & $1(0.3)$ & $3(2.5)$ & \\
\hline Professional status & & & & 0.321 \\
\hline Employee & $51(11.6)$ & $33(10.3)$ & $18(15)$ & \\
\hline Self employed & $12(2.7)$ & $7(2.2)$ & $5(54.2)$ & \\
\hline Inactive & $375(85)$ & $279(86.9)$ & $96(78)$ & \\
\hline Retirement & $3(0.7)$ & $2(0.6)$ & $1(0.8)$ & \\
\hline
\end{tabular}

* The Chi-square test (x2) or Fisher's exact test; HADS: Hospital Anxiety and Depressive scale 
Table 1 (continued). Socio-demographic and psychological characteristics of the patients classified according to the presence of chronic pain

\begin{tabular}{|c|c|c|c|c|}
\hline Variable & $\begin{array}{c}\text { All patients } \\
\mathrm{N}(\%)\end{array}$ & $\begin{array}{c}\text { Chronic pain } \\
\mathrm{N}(\%)\end{array}$ & $\begin{array}{c}\text { No Chronic pain } \\
N(\%)\end{array}$ & $P$ value \\
\hline Marital status & & & & $<0.01^{\star}$ \\
\hline Single & $61(13.8)$ & $33(7.5)$ & $28(23.3)$ & \\
\hline Married & $297(67.3)$ & 218 (67.9) & $79(65.8)$ & \\
\hline Divorced & $7(1.6)$ & $5(1.6)$ & $2(1.7)$ & \\
\hline Widower & $76(17.2)$ & $65(20.2)$ & $11(9.2)$ & \\
\hline \multicolumn{5}{|l|}{ Living conditions } \\
\hline living alone & $270(61.2)$ & $161(50.2)$ & $11(9.2)$ & $<0.01^{\star}$ \\
\hline living with others & $171(38.8)$ & $160(49.8)$ & $109(90.8)$ & \\
\hline \multicolumn{5}{|l|}{ Health coverage } \\
\hline With assurance & 431 (97.9) & $311(96.88)$ & $120(100)$ & $0.040^{\star}$ \\
\hline Without assurance & $10(2.3)$ & $10(0.03)$ & 0 & \\
\hline \multicolumn{5}{|l|}{ Anxiety } \\
\hline Yes (Score HADS $\geq 11$ ) & $300(68)$ & 267 (83.2) & $33(16.8)$ & $<0.01^{\star}$ \\
\hline No (Score HADS < 11) & $41(32)$ & $54(16.8)$ & $87(72.5)$ & \\
\hline \multicolumn{5}{|l|}{ Depression } \\
\hline Yes (Score HADS $\geq 11$ ) & $291(66)$ & $258(80.4)$ & $33(27.5)$ & $<0.01^{\star}$ \\
\hline No (Score HADS <11) & $150(34)$ & $63(19.6)$ & $87(79.2)$ & \\
\hline Use of anxiety medications & $12(2.7)$ & $10(3.1)$ & $2(1.7)$ & 0.322 \\
\hline Use of depression medications & $10(2.3)$ & $8(2.5)$ & $2(1.7)$ & 0.280 \\
\hline
\end{tabular}

${ }^{\star}$ The Chi-square test (x2) or Fisher's exact test; HADS: Hospital Anxiety and Depressive scale

Table 2. Clinical and diyalitic characteristics of the patients classified according to the presence of chronic pain

\begin{tabular}{|c|c|c|c|c|}
\hline Variable & $\begin{array}{c}\text { All patients } \\
\mathrm{N}(\%)\end{array}$ & $\begin{array}{c}\text { Chronic pain } \\
\mathrm{N}(\%) \\
\end{array}$ & $\begin{array}{c}\text { No chronic pain } \mathbf{N} \\
(\%)\end{array}$ & $P$ value \\
\hline Respect of hygieno-dietetic rules & $10(2.3)$ & $8(2.5)$ & $2(1.7)$ & 0.459 \\
\hline Toxic Habits & $17(3.9)$ & $17(5.3)$ & $0(0)$ & 0.040 \\
\hline Body Mass Index (Kg/m2) & $23.18 \pm 3.6$ & $23.15 \pm 3.53$ & $23.24 \pm 3.77$ & 0.798 \\
\hline$<18.5$ & $74(16.8)$ & $52(16.2)$ & $22(18.3)$ & \\
\hline $18.5-24.9$ & $241(54.6)$ & $175(54.5)$ & $66(55)$ & \\
\hline 25-29.9 & $126(28.8)$ & $94(29.3)$ & $32(26.7)$ & \\
\hline \multicolumn{5}{|l|}{ Co-morbidity } \\
\hline Hypertension & $235(53.3)$ & $177(55.1)$ & $58(48.3)$ & 0.121 \\
\hline Diabetes mellitus & $165(37.4)$ & $129(40.2)$ & $36(30)$ & 0.031 \\
\hline Cardiovascular diseases & $19(4.3)$ & $16(5)$ & $3(2.5)$ & 0.192 \\
\hline System diseases & $4(0.9)$ & $3(0.9)$ & $1(0.8)$ & 0.279 \\
\hline Cancer & $4(0.9)$ & $3(0.7)$ & $1(0.2)$ & 0.701 \\
\hline Liver diseases & $3(0.7)$ & $2(0.6)$ & $1(0.8)$ & 0.615 \\
\hline A prior stroke history & $32(7.3)$ & $26(8.1)$ & $6(1.4)$ & 0.183 \\
\hline \multicolumn{5}{|l|}{ Causal Nephropathy } \\
\hline Indeterminate nephropathy & $147(33.3)$ & $92(28.7)$ & $55(45.8)$ & $<0,01^{*}$ \\
\hline Diabetic nephropathy & $180(40.8)$ & $143(44.5)$ & $37(30.8)$ & $0,006^{\star}$ \\
\hline Glomerular chronic & $42(9.5)$ & $32(10)$ & $10(8.3)$ & 0,375 \\
\hline Vascular Nephropathy & $29(6.6)$ & $25(7.8)$ & $4(3.3)$ & 0,066 \\
\hline Polycystic kidney disease & $13(2.9)$ & $10(3.1)$ & $3(2.5)$ & 0,509 \\
\hline Tubulo-interstitial nephritis & $20(4.5)$ & $13(4)$ & $7(5.8)$ & 0,285 \\
\hline Eclampsia & $4(0.9)$ & $2(0.6)$ & $2(1.7)$ & 0,299 \\
\hline \multicolumn{5}{|l|}{ Renal therapy replacement } \\
\hline Hemodialysis & $441(100)$ & $321(100)$ & $120(100)$ & 0.273 \\
\hline Interdialytic Weight Gain /Kg & $2.38 \pm 1.05$ & $2.46 \pm 1.06$ & $2.17 \pm 0.98$ & 0.233 \\
\hline$<1$ & 205 (46.5) & $146(45.5)$ & $59(49.2)$ & \\
\hline $1-2$ & $155(35.1)$ & $120(37.4)$ & $35(29.2)$ & \\
\hline$>2$ & $81(18.4)$ & $55(17.1)$ & $26(21.7)$ & \\
\hline Duration on hemodialysis /mouths & $64.84 \pm 49.67$ & $65.64 \pm 49.59$ & $62.71 \pm 50.02$ & 0.152 \\
\hline$<50$ & $208(47.2)$ & $147(45.8)$ & $61(50.8)$ & \\
\hline $50-100$ & $152(34.5)$ & $119(37.1)$ & $33(27.5)$ & \\
\hline$>100$ & $81(18.4)$ & $55(17.1)$ & $26(21.7)$ & \\
\hline \multicolumn{5}{|l|}{ Number of dialysis sessions / week } \\
\hline 2 Sessions & 399 (90.5) & $302(94.1)$ & $97(80.8)$ & $<0,01^{\star}$ \\
\hline 3 Sessions & $42(9.5)$ & $19(5.9)$ & $23(19.2)$ & \\
\hline \multicolumn{5}{|l|}{ Vascular Access } \\
\hline AVF proximal & $131(29.7)$ & $100(31.2)$ & $31(25.8)$ & 0.273 \\
\hline AVF distal & $293(66.4)$ & $211(65.7)$ & $82(68.3)$ & \\
\hline Tunneled jugular catheter & $17(3.9)$ & $10(3.1)$ & $7(5.8)$ & \\
\hline
\end{tabular}

* The Chi-square test (x2) or Fisher's exact test

chronic renal failure (ESRD) and the list of recorded comorbidities are summarized in Table 2 . Approximately 90.5
$\%$ of patients ( $n=399 / 441$ ) had a frequency of 2 sessions of 4 to 4.5 hours dialysis per week, while the remaining 42 (9.5\%) were 
Table 3. Biological characteristics of the patients classified according to the presence of chronic pain

\begin{tabular}{|c|c|c|c|c|}
\hline Variable & $\begin{array}{c}\text { All patients } \\
\mathrm{N}(\%) \\
\end{array}$ & $\begin{array}{c}\text { Chronic pain } \\
\mathrm{N}(\%) \\
\end{array}$ & $\begin{array}{c}\text { No chronic pain } \\
\mathrm{N}(\%) \\
\end{array}$ & $P$ value \\
\hline Hemoglobin (g/dl) & $9.65 \pm 1.58$ & $9.72 \pm 1.54$ & $9.46 \pm 1.67$ & 0.196 \\
\hline$<8$ & $51(11.6)$ & $34(10.6)$ & $17(14.2)$ & \\
\hline $8-10,9$ & $289(65.5)$ & 207 (64.5) & $171(66.8)$ & \\
\hline$\geq 11$ & $101(22.9)$ & $80(24.9)$ & $21(17.5)$ & \\
\hline PTH (mg/ml) & $476.17 \pm 216.97$ & $482.90 \pm 216.94$ & $458.17 \pm 216.91$ & 0.884 \\
\hline$<300$ & $136(30.8)$ & $99(30.8)$ & $37(30.8)$ & \\
\hline $300-600$ & $214(48.5)$ & $154(48)$ & $60(50)$ & \\
\hline$>600$ & $91(20.6)$ & $68(21.2)$ & 23 (19.2) & \\
\hline $\mathrm{Ca}(\mathrm{mmol} / \mathrm{l})$ & $51.05 \pm 31.46$ & $49.53 \pm 30.08$ & $55.12 \pm 32.74$ & $0.022^{\star}$ \\
\hline$<90$ & $393(89.1)$ & $294(91.6)$ & $99(82.5)$ & \\
\hline $90-105$ & $40(9.1)$ & $22(6.9)$ & $18(15)$ & \\
\hline$>105$ & $8(1.8)$ & $5(1.6)$ & $3(2.5)$ & \\
\hline P04(mg/l) & $46.83 \pm 16.48$ & $46.99 \pm 15.78$ & $46.43 \pm 18.28$ & 0.133 \\
\hline$<25$ & $17(3.9)$ & $9(2.8)$ & $8(6.7)$ & \\
\hline $25-45$ & $247(56)$ & $185(57.6)$ & $62(51.7)$ & \\
\hline$>45$ & $177(40.1)$ & $127(39.6)$ & $50(41.7)$ & \\
\hline Albumin (g/l & $42.75 \pm 14.68$ & $42.78 \pm 14.52$ & $42.66 \pm 15.18$ & 0.884 \\
\hline$<38$ & $136(30.8)$ & $99(30.8)$ & $37(30.8)$ & \\
\hline $38-50$ & $214(48.5)$ & $154(48)$ & $60(50)$ & \\
\hline$>50$ & $91(20.6)$ & $68(21.2)$ & 23 (19.2) & \\
\hline CRP (mg/l) & $63.27 \pm 28.34$ & $64.85 \pm 28.38$ & $59.05 \pm 27.91$ & 0.321 \\
\hline$<6$ & $51(11.6)$ & 33 (10.3) & $18(15)$ & \\
\hline $6-50$ & $12(2.7)$ & 7 (2.2) & $5(4.2)$ & \\
\hline $50-100$ & $375(85)$ & 279 (86.9) & $96(80)$ & \\
\hline$>100$ & $3(0.7)$ & $2(0.6)$ & $1(0.8)$ & \\
\hline
\end{tabular}

${ }^{\star}$ The Chi-square test $(\mathrm{x} 2)$ or Fisher's exact test

dialyzed 3 times per week for 4 hours per session. The mean inter-dialytic weight gain (IDWG) was 2.38 (1.05) kg. Table 2 summarizes all of the clinical and dialytic parameters collected, the etiologies of end-stage chronic renal failure (ESRD) and the list of recorded comorbidities. Table 3 shows the biological characteristics of the sample.

\section{Prevalence of Chronic Pain}

Of the 441 patients, 321 reported the presence of chronic pain (CP), for a prevalence of $72.8 \%$. In the group with chronic pain $(\mathrm{CP}+)$ : The mean age is $57.04(15.28)$, the sex ratio is $174 \mathrm{H}$ / 147F, 206 patients (64.2\%) were illiterate, 279 (86.9\%) were unemployed, the mean duration of dialysis was $65.64(49.59)$ months (see Table 1). There were 267 (80.4\%) who selfreported anxiety and 211 (65.7\%) depression.

\section{Characteristics of Chronic Pain}

Chronic pain intensity was absent, mild, moderate, severe and unbearable in 1 (0.3\%), 69 (21.5\%), 110 (34.3\%), 131 (40.8 $\%)$, and $10(3.1 \%)$ of patients respectively. Its frequency was mostly intermittent $(61.7 \%)$. However, pain was only worsened during dialysis sessions in $9.6 \%$ of cases.

The chronic pain described by the patients was in the lowers extremities in $39.9 \%$ of the cases, in the back in $19.3 \%$, in the head in $14 \%$, in the abdomen in $11.2 \%$, multifocal in 10 $\%$, in the shoulders in $5.6 \%$, and with an average number of painful sites per patient of 4.41 (1.24). Thus, the identified causes of chronic CP are the following: osteoarticular, neurological, vascular, digestive and post-traumatic in respectively 155 (48.3\%), 83 (25.9\%), 45 (14\%), 35 (10.9\%), 3 (0.9\%) (See Table 4).

\section{Analgesic Admission}

We will retain 191 patients, approximately (59.9\%), had taking analgesics and up to $59.9 \%$ of patients taking analgesics
Table 4. Characteristics of chronic pain and Analgesic admission

\begin{tabular}{lc}
\hline Variable & Frequency N (\%) \\
\hline Intensity of chronic pain & \\
Absent: VAS at 0 & $1(0.3)$ \\
Mild: VAS from 1 to 3 & $69(21.5)$ \\
Moderate: VAS from 4 to 6 & $110(34.3)$ \\
Severe: VAS from 7to 9 & $131(40.8)$ \\
Unbearable: VAS at 10 & $10(3.1)$ \\
Pain frequency & \\
$\quad$ Intermittent & $198(61.7)$ \\
Daily & $73(22.7)$ \\
Permanent & $46(14.3)$ \\
Rare & $4(1.2)$ \\
Causes of chronic pain & \\
Osteo-articular & $155(48.3)$ \\
Neurologic & $83(25.9)$ \\
Vascular & $45(14)$ \\
Digestive & $35(10.9)$ \\
Post-traumatic & $3(0.9)$ \\
Painful region & \\
Head & $45(14)$ \\
Shoulders & $18(5.6)$ \\
Abdomen & $36(11.2)$ \\
Back & $62(19.3)$ \\
Lower limb & $128(39.9)$ \\
Multifocal pain & $32(10)$ \\
\hline VAS: Visual ana
\end{tabular}

VAS: visual analog scale

frequently in $74.3 \%$ of cases, daily in $23.6 \%$ of cases and rarely in $2.4 \%$ of cases. $92.1 \%$ of analgesics are level 1 and $7.9 \%$ are level 2 and no level 3 analgesic is used. This admission only allowed complete relief in $6 \%$ of cases and the degree of relief remains low in 131 patients (68.6\%). The consequent analgesic admission is summarized in Table 4. 
Table 4 (continued). Characteristics of chronic pain and Analgesic admission

\begin{tabular}{lc}
\hline Variable & Frequency N (\%) \\
\hline Analgesic admission & $191(59.9)$ \\
Level 1 & $176(92.1)$ \\
Level 2 & $15(7.9)$ \\
Level 3 & 0 \\
Rhythm analgesic & \\
Frequent & $142(74.3)$ \\
Daily & $45(23.6)$ \\
Rare & $4(2.1)$ \\
Relief after taking analgesics & $2(1)$ \\
No relief & $17(8.9)$ \\
low & $103(53.9)$ \\
Moderate & $63(33)$ \\
Important & $6(3.1)$ \\
Complete & \\
Dependence on analgesic & $45(23.6)$ \\
\hline
\end{tabular}

\section{Impact of Chronic Pain}

The impact of chronic pain on the daily life of patients is summarized in Table 5. Note that it completely interfered with general activity, with the ability to walk and usual work in 234 patients (72.9\%), 221 (68.85\%), 230 (71.7\%) respectively. Regarding the mood, the relations with others and the enjoyment of life, chronic pain is completely embarrassing 69.8 $\%$ (224 patients), $34 \%$ (109 patients) and $34.6 \%$ (111 patients) of cases respectively.

\section{Factors Associated with Chronic Pain \\ Univariate analysis}

1. Socio-demographic and psychological factors on univariate analysis (See Table 6)

2. Clinical factors on univariate analysis (See Table 7)

3. Biological and dialytic factors on univariate analysis (See Table 8)

Table 5. Impact of chronic pain

\begin{tabular}{|c|c|c|c|c|c|}
\hline & $\begin{array}{c}\text { No gene } \\
\text { N (\%) }\end{array}$ & $\begin{array}{c}\text { Weak gene } \\
\mathrm{N}(\%)\end{array}$ & Moderate gene $\mathbf{N}(\%)$ & $\begin{array}{c}\text { Important gene } \mathbf{N}( \\
\%)\end{array}$ & $\begin{array}{c}\text { Complet gene } \mathrm{N} \\
(\%)\end{array}$ \\
\hline General activity & 0 & $2(0,6)$ & $14(4,4)$ & $71(22,1)$ & $234(72,9)$ \\
\hline Ability to walk & 0 & $1(0,3)$ & $21(6,5)$ & $78(24,3)$ & $221(68,8)$ \\
\hline Usual Work & 0 & $1(0,3)$ & $19(5,9)$ & $71(22,1)$ & $230(71,7)$ \\
\hline Sleep & $1(0,3)$ & $7(2,2)$ & $93(29)$ & $80(24,9)$ & $140(43,6)$ \\
\hline Mood & 0 & $2(0,6)$ & $53(16,5)$ & $42(13,1)$ & $224(69,8)$ \\
\hline Relations with others people & 0 & $13(4)$ & $140(31,7)$ & $59(18,4)$ & 109(34) \\
\hline Enjoyment of life & 0 & $8(2,5)$ & $122(38)$ & $80(24,9)$ & $111(34,6)$ \\
\hline
\end{tabular}

Table 6. Socio-demographic and psychological factors associated with chronic pain on univariate analysis

\begin{tabular}{|c|c|c|c|}
\hline Variable & OR & Cl 95\% & $P$ value \\
\hline \multicolumn{4}{|l|}{ Age } \\
\hline [18-45 years] & 2.34 & $1.33-4.12$ & $<0.01$ \\
\hline [46-65 years] & 1.30 & $0.76-2.20$ & 0.329 \\
\hline$>65$ years & 1 & / & / \\
\hline \multicolumn{4}{|l|}{ Sexe } \\
\hline Male & 1.40 & $0.91-2.16$ & 0.119 \\
\hline Female & 1 & / & l \\
\hline \multicolumn{4}{|l|}{ Level of studies } \\
\hline Illiterate & 0.12 & $0.01-1.18$ & 0.070 \\
\hline Primary & 0.09 & $0.01-1.00$ & 0.050 \\
\hline Middle school & 0.29 & $0.02-3.14$ & 0.314 \\
\hline Hight school & 0.06 & $0.00-0.76$ & 0.029 \\
\hline Higher education & 1 & / & / \\
\hline \multicolumn{4}{|l|}{ Professional status } \\
\hline Employee & 1.09 & $0.09-12.87$ & 0.945 \\
\hline Self employed & 1.71 & $0.10-20.43$ & 0.793 \\
\hline Inactive (unemployed) & 0.68 & $0.06-7.67$ & 0.761 \\
\hline Retirement & 1 & I & / \\
\hline \multicolumn{4}{|l|}{ Marital status } \\
\hline Single & 5.01 & $2.22-11.31$ & $<0.01$ \\
\hline Married & 2.14 & $1.07-4.26$ & 0.030 \\
\hline Divorced & 2.36 & $0.40-13.73$ & 0.338 \\
\hline Widower & 1 & l & / \\
\hline \multicolumn{4}{|l|}{ Living conditions } \\
\hline Living alone & 9.84 & $5.10-19.00$ & $<0.01$ \\
\hline Living with others & 1 & l & / \\
\hline \multicolumn{4}{|l|}{ Health coverage } \\
\hline With assurance & 0.17 & $0.03-0.83$ & 0.99 \\
\hline Without assurance & 1 & / & l \\
\hline \multicolumn{4}{|l|}{ Anxiety } \\
\hline Yes (Score HADS $\geq 11$ ) & 13.03 & $7.93-21.40$ & $<0.01$ \\
\hline No (Score HADS <11) & 1 & 1 & / \\
\hline \multicolumn{4}{|l|}{ Depression } \\
\hline Yes (Score HADS $\geq 11$ ) & 10.79 & $6.63-17.55$ & $<0.01$ \\
\hline No (Score HADS <11) & 1 & 1 & / \\
\hline
\end{tabular}


Table 7. Clinical factors associated with chronic pain on univariate analysis

\begin{tabular}{|c|c|c|c|}
\hline Variable & OR & $\mathrm{Cl} 95 \%$ & $P$ value \\
\hline \multicolumn{4}{|c|}{ Respect hygieno-dietetic rules } \\
\hline Yes & 0.89 & $0.09-8.64$ & 0.921 \\
\hline No & 1 & l & l \\
\hline \multicolumn{4}{|l|}{ Toxic Habits } \\
\hline Yes & 0.16 & $0.03-0.17$ & 0.998 \\
\hline No & 1 & l & l \\
\hline \multicolumn{4}{|c|}{ Body Mass Index (kg/m²) } \\
\hline$<18.5$ & 0.48 & $0.65-2.35$ & 0.506 \\
\hline $18.5-24.9$ & 0.32 & $0.67-1.81$ & 0.683 \\
\hline $25-29.9$ & 1 & 1 & / \\
\hline \multicolumn{4}{|l|}{ Hypertension } \\
\hline Yes & 1.31 & $0.86-2.00$ & 0.203 \\
\hline No & 1 & 1 & l \\
\hline \multicolumn{4}{|c|}{ Diabetes mellitus } \\
\hline Yes & 0.63 & $0.40-1.00$ & 0.050 \\
\hline No & 1 & 1 & / \\
\hline \multicolumn{4}{|c|}{ Cardiovascular diseases } \\
\hline Yes & 0.48 & $0.14-1.70$ & 0.262 \\
\hline No & 1 & 1 & l \\
\hline \multicolumn{4}{|c|}{ Systemic diseases } \\
\hline Yes & 0.000 & $0.00-0.71$ & 0.999 \\
\hline No & 1 & 1 & 1 \\
\hline \multicolumn{4}{|l|}{ Cancer } \\
\hline Yes & 0.89 & $0.09-8.64$ & 0.921 \\
\hline No & 1 & 1 & / \\
\hline \multicolumn{4}{|l|}{ Liver diseases } \\
\hline Yes & 1.34 & $0.12-23.13$ & 0.812 \\
\hline No & 1 & l & l \\
\hline \multicolumn{4}{|c|}{ A prior stroke history } \\
\hline Yes & 0.59 & $0.24-1.48$ & 0.269 \\
\hline No & 1 & / & / \\
\hline \multicolumn{4}{|c|}{ indeterminate nephropathy } \\
\hline Yes & 2.10 & $1.36-3.24$ & $<0.01$ \\
\hline No & 1 & / & / \\
\hline \multicolumn{4}{|c|}{ Diabetic nephropathy } \\
\hline Yes & 0.55 & $0.35-0.86$ & 0.010 \\
\hline No & 1 & 1 & l \\
\hline \multicolumn{4}{|c|}{ Glomerular chronic nephropathy } \\
\hline Yes & 0.82 & $0.39-1.72$ & 0.603 \\
\hline No & 1 & 1 & / \\
\hline \multicolumn{4}{|c|}{ Vascular Nephropathy } \\
\hline Yes & 0.40 & $0.13-1.19$ & 0.103 \\
\hline No & 1 & / & / \\
\hline \multicolumn{4}{|c|}{ Polycystic kidney disease } \\
\hline Yes & 0.79 & $0.21-2.94$ & 0.734 \\
\hline No & 1 & I & / \\
\hline \multicolumn{4}{|c|}{ Tubulo-interstitial nephritis } \\
\hline Yes & 0.57 & $0.57-3.77$ & 0.426 \\
\hline No & 1 & / & / \\
\hline \multicolumn{4}{|l|}{ Eclampsia } \\
\hline Yes & 2.70 & $0.37-19.41$ & 0.323 \\
\hline No & 1 & 1 & 1 \\
\hline
\end{tabular}

Table 8. Biological and dialytic factors associated with chronic pain on univariate analysis

\begin{tabular}{|c|c|c|c|}
\hline Variable & OR & $\mathrm{Cl} 95 \%$ & $P$ value \\
\hline \multicolumn{4}{|c|}{$\begin{array}{l}\text { Dialytic parameters } \\
\text { Interdialytic Weight Gain (IDWG) }\end{array}$} \\
\hline$<1 \mathrm{~kg}$ & 0.85 & $0.49-1.49$ & 0.580 \\
\hline $1-2 \mathrm{Kg}$ & 0.61 & $0.33-1.12$ & 0.114 \\
\hline$>2 \mathrm{Kg}$ & 1 & / & / \\
\hline \multicolumn{4}{|c|}{ Duration on hemodialysis /mouths } \\
\hline$<50$ & 0.87 & $0.50-1.52$ & 0.645 \\
\hline $50-100$ & 0.58 & $0.32-1.07$ & 0.084 \\
\hline$>10$ & 1 & / & / \\
\hline \multicolumn{4}{|c|}{ Number of dialysis sessions / week } \\
\hline 2 Sessions & 0.26 & $0.13-0.50$ & $<0.01$ \\
\hline 3 Sessions & 1 & / & / \\
\hline
\end{tabular}

OR: odds ratio, $\mathrm{Cl}$ : confidence interval 
Table 8 (continued). Biological and dialytic factors associated with chronic pain on univariate analysis

\begin{tabular}{|c|c|c|c|}
\hline Variable & OR & $\mathrm{Cl} 95 \%$ & $P$ value \\
\hline \multicolumn{4}{|l|}{ Vascular Access } \\
\hline AVF proximal & 0.44 & $0.15-1.26$ & 0.127 \\
\hline AVF distal & 0.55 & $0.20-1.50$ & 0.248 \\
\hline Tunneled jugular catheter & 1 & I & / \\
\hline \multicolumn{4}{|l|}{ Hematology parameters } \\
\hline \multicolumn{4}{|l|}{ Hemoglobin (g/dl) } \\
\hline$<8$ & 1.90 & $0.89-4.05$ & 0.094 \\
\hline $8-10.9$ & 1.50 & $0.87-2.60$ & 0.138 \\
\hline$\geq 11$ & 1 & / & / \\
\hline \multicolumn{4}{|l|}{ PTH (pg/ml) } \\
\hline$<300$ & 1.10 & $0.60-2.02$ & 0.746 \\
\hline $300-600$ & 1.15 & $0.65-2.01$ & 0.320 \\
\hline$>600$ & 1 & l & l \\
\hline \multicolumn{4}{|l|}{$\mathrm{Ca}(\mathrm{mmol} / \mathrm{l})$} \\
\hline$<90$ & 0.56 & $0.13-2.39$ & 0.435 \\
\hline $90-105$ & 1.36 & $2.28-6.49$ & 0.697 \\
\hline$>105$ & 1 & l & / \\
\hline \multicolumn{4}{|l|}{ P04(mg/l) } \\
\hline$<25$ & 2.25 & $0.82-6.18$ & 0.113 \\
\hline $25-45$ & 0.85 & $0.551-1.31$ & 0.469 \\
\hline$>45$ & 1 & / & / \\
\hline \multicolumn{4}{|l|}{ Albumin (g/l) } \\
\hline$<38$ & 1.10 & $0.60-2.02$ & 0.746 \\
\hline $38-50$ & 1.15 & $0.65-2.01$ & 0.620 \\
\hline$>50$ l & 1 & / & / \\
\hline \multicolumn{4}{|l|}{ CRP (mg/l) } \\
\hline$<6$ & 1.09 & $0.09-12.87$ & 0.945 \\
\hline $6-50$ & 1.42 & $0.10-20.43$ & 0.793 \\
\hline $50-100$ & 0.68 & $0.06-7.67$ & 0.761 \\
\hline$>100$ & 1 & / & / \\
\hline
\end{tabular}

OR: odds ratio, $\mathrm{Cl}$ : confidence interval

Table 9. Factors associated with chronic pain on multivariate analysis

\begin{tabular}{|c|c|c|c|}
\hline Variable & aOR & $\mathrm{Cl} 95 \%$ & $P$ value \\
\hline \multicolumn{4}{|l|}{ Level of studies } \\
\hline Hight school & 0.01 & $0.00-0.28$ & $<0.001$ \\
\hline \multicolumn{4}{|l|}{ Marital status } \\
\hline Single & 8.37 & $1.40-49.83$ & 0.020 \\
\hline \multicolumn{4}{|l|}{ Living conditions } \\
\hline Alone & 24.04 & $8.46-68.32$ & $<0.001$ \\
\hline \multicolumn{4}{|l|}{ Anxiety } \\
\hline Yes & 19.91 & $8.17-48.50$ & $<0.001$ \\
\hline \multicolumn{4}{|l|}{ Depression } \\
\hline yes & 20.74 & $9.13-17.10$ & $<0.001$ \\
\hline
\end{tabular}

aOR: Adjusted odds ratio, $\mathrm{Cl}$ : confidence interval

\section{Multivariate analysis}

The variables that were included in the regression equation were: age, sexe, education level, marital status, living conditions, duration of hemodialysis, inter-dialytic weight gain, Number of dialysis sessions per week, comorbidities (hypertension, diabetes and cardiovascular diseases), Haemoglobin, Calcemia, indeterminate nephropathy, Diabetic nephropathy, Vascular Nephropathy, vascular access, anxiety and depression.

The results of the multivariate analysis revealed, among all the variables, five factors which had a strong statistical correlation $(P<.05)$ with chronic pain in hemodialysis patients: depression, anxiety, education level, marital status and living conditions (See Table 9).

\section{DISCUSSION}

\section{Prevalence of Chronic Pain}

The prevalence of chronic pain in this study is $72.8 \%$, it is almost similar to $70.9 \%$ in the study of Elharraqui [10], $74.4 \%$ in Sadigova's work [5], 82 \% in the Flesherman series [3], while in other series [11-13], this rate does not exceed $50.1 \%$. This difference may be justified by the difference between the contexts of the assessment and management approaches of chronic pain, but also by the differences in the perception of pain in different countries, different cultures and different ethnicities. Several studies have reported that there are large and complex cultural variations between countries and ethnicities in the prevalence and outcomes of pain-related conditions $[14,15]$, another study showed that the prevalence of chronic pain and its disability is more important in underdeveloped countries than in developed ones [16]. 


\section{Chronic Pain Assessment}

In this study, the identified causes of chronic pain are as follows: osteoarticular, neurological, vascular, digestive and post-traumatic in respectively 155 (48.3\%), 83 (25.9\%), 45 (14 $\%), 35$ (10.9\%), 3 (0.9\%). In fact, $82 \%$ of chronic kidney disease patients undergoing dialysis have been reported to have chronic pain [11] due to the frequent osteoarticular, cardiovascular, digestive, and traumatic complications in this population [17]. The main cause of chronic pain in our patients is osteoarticular $(48.3 \%)$, as has reported in some trials $[13,18]$. The pain associated with osteoarticular complications in chronic dialysis patients can be explained by ß2-microglobulin amyloidosis: The presence of amyloid deposits fibrillar mainly in joint tissues and in bones, clinically causes the appearance of osteoarticular pain syndromes [19]. Therefore, early and optimal management of disorders bone mineral linked to Chronic Renal Insufficiency was expected to reduce in part of the occurrence of these difficulties [13]. In our cases, the intensity of the pain was significant with $40.8 \%$ of severe form versus $44 \%$ in the Bouaatar study [12], and $53.3 \%$ in the BenBassat study [20]. Multiple studies indicated a high prevalence of patients with moderate or severe pain [4]. The most common painful site was in the lower limbs in $39.9 \%$ of patients, as reported in other studies $[5,13,20]$, the pain was only worsened during dialysis sessions in $9.6 \%$ of cases.

\section{Analgesic Admission}

Although more than half ( $59.5 \%$ ) of patients had regular use of level 1 and level 2 analgesics, complete relief was only noted in $3.1 \%$ of patients. Results of several studies show insufficient relief of Chronic Pain in hemodialysis patients with analgesics [5]. This finding may be justified by the lack of knowledge of the exact mechanism of Chronic Pain for an adequate prescription of analgesics or opioids in chronic hemodialysis patients [21]. However, special considerations should be taking in hemodialysis patients to minimize direct renal complications induced by analgesics and other complications related to drug accumulation due to reduced renal clearance [22].

In addition, studies prove that drug treatment remains insufficient in face of chronic pain in hemodialysis patients. $[23,24]$. In this regard, other non-pharmacological treatments could improve the pain, depression, anxiety, functionality and quality of life of hemodialysis patients with chronic pain without a major adverse event [24]. It has been proven that there are non-drug analgesic approaches that can alleviate chronic pain in hemodialysis patients such as conscious calming gestures [23], cognitive behavioral therapies [2], and hypnosis: several local experiences have highlighted the benefits of hypnosis for controlling acute and chronic pain in hemodialysis patients [25].

\section{Impact of Chronic Pain on Daily Life}

In this study, Chronic pain caused complete discomfort in general activity in $72.9 \%$ of cases versus $62.1 \%$ in the study by El Harraqui [10], on the ability to walk in $68.85 \%$ of cases. This rate varies from $19.1 \%$ to $44.1 \%$ in other studies [12, 13]. Also, usual work is completely hampered by chronic pain in $71.7 \%$ of cases. Indeed, several authors have reported that chronic pain disrupts body patterns, reduces overall activity level, and causes intolerance to physical and/or intellectual effort $[2,26]$, chronic pain is, therefore, a source of disability, and major alterations in daily life [2]. Furthermore, due to chronic pain complete sleep discomfort was noted in $38.01 \%$ of cases, several studies prove that chronic pain is significantly associated with sleep disorders and even insomnia in hemodialysis patients $[11,18,27,28]$. Chronic pain is a multidimensional phenomenon that has several components: physical, psychological, and social. If left untreated, it can affect several aspects: mood, enjoyment of life, and relationship with others [11]. In our study, these aspects are completely affected in $69.80 \%, 34.6 \%$ and $34 \%$ respectively. Chronic pain can therefore only be evaluated in a relevant way if it is listened to, observed, but also analyzed through the repercussions that are personal, physical, and psychological, as well as social and professional [26].

\section{Factors Associated with Chronic Pain}

Multivariate analysis in our study showed a strong correlation between chronic pain and five factors: depression, anxiety, education level, marital status and living conditions. For the first two factors, several authors have reported that the comorbidity of depression and anxiety are common in chronically painful people, and people with chronic pain are more likely to have depressive and anxious symptoms than people without pain $[29,30]$

Several investigators have estimated that depression occurs in about $20 \%$ to $67 \%$ of dialysis patients [31-34]. This variation would be linked to the use of tools and different methodologies [35]. In our study, the prevalence of depression is $66 \%$ in the whole population, and $80.4 \%$ in the group of chronic pain sufferers, this finding is explained by the causal relationship between depression and chronic pain, several authors have proven that the causal link between chronic pain and depression is bilateral: chronic pain depresses the patient and this depression promotes chronic pain $[36,37]$. Besides depression, anxiety, is the most prevalent psychological factor associated with chronic pain [38]. A recent study places the prevalence of anxiety in chronic hemodialysis patients at 36.9 $\%$ [34], in our study, this rate represents $68 \%$ for the entire population and $83.2 \%$ in the group of chronic pain sufferers.

Studies have shown that anxiety about health is greater in people with pain than in the control group [39]. Along with these studies, many other studies have demonstrated the importance of psychological factors in the management of chronic pain [2]. In our study, the education level is retained also as a factor associated with chronic pain, people with little education are more likely to suffer from chronic pain than those with a higher level of education [1]. Indeed, an advanced level of education protects against chronic pain in hemodialysis patients, probably because of due to the cognitive skills that allow them to accept and manage their pain. These skills based on the level of education allow the patient to observe chronic pain, and understand it and thus to appropriate it [40]. Similarly, illiteracy is identified as a risk factor linked to chronic pain in hemodialysis [10].

The social status is also retained as a factor associated with chronic pain. Loneliness and social isolation increase the perception of chronic pain. Several studies confirm that being integrated into a social network provides support for the painful ordeal and promotes the reduction of the perceived chronic pain. Also, family support positively influences the patient's behavioral and attitude responses to chronic pain. $[40,41]$ On the other way, disappointment in relation to the social support received, favors passive strategies for coping 
with pain, increases the negative emotional response to pain; adjustment to chronic pain is therefore less good [38].

In our results, the fifth factor retained in multivariate analysis is marital status. Multiple studies have explored the potential association between marital status and chronic pain in hemodialysis patients [40-42]. One of these studies, found no correlation between pain and marital status [43], while the others confirmed that marital status is significantly associated with chronic pain on hemodialysis [40,41]. In our study, unmarried patients ultimately reported more chronic pain, similar to the work of Binik [43]. In fact, the understanding of the spouse and his real support are preponderant factors, for the chronic pain patient which condition, in a positive way, all his other behavioral responses and his attitudes towards pain [40].

\section{STRENGTHS AND LIMITATIONS}

Strengths of our study include its multi-center environment and relatively large sample size. It was also the first study in this region to assess chronic pain, examine its effect on the daily life of hemodialysis patients, and study the social, demographic, clinical and psychological factors associated with it. In addition, the current study used the BPI and HADS scales which are widely accepted assessment tools to study chronic pain symptoms and diagnose depression and anxiety, respectively, in hemodialysis patients. However, this study had some limitations,

The first is that the patients came from a single geographic region; It was conducted in a single population of hemodialysis patients and may not be generalizable to other hemodialysis populations. The second is that the cause of the pain has been determined just by examination of files and the last, is that the confidence intervals of the Odds rations are wide, it shows that the evaluation of the parameters is not so precise due to the size of the sample.

However, we believe that the results provide a solid basis for studies that will further explore this aspect. The ultimate goal should be a better understanding and treatment of chronic pain in hemodialysis patients.

\section{CONCLUSION}

Chronic pain on hemodialysis needs to be understood in the context of social, biological, psychological, and physical factors. A multidisciplinary approach is recommended for the management of complex pain syndromes, include both pharmacological and non-pharmacological interventions and that involves nurses, physiotherapists, occupational therapists, psychologists, health educators and family, to ensure a good quality of life for this vulnerable population of hemodialysis patients.

Pain assessment and management need to be incorporated into standard care for these patients.

However, additional research is required to identify the most effective approaches to pain management for long-term hemodialysis patients.

Funding: No funding source is reported for this study.

Acknowledgements: The author would like to thank the staff of the public hemodialysis centers in the Souss Massa region for the collaboration throughout the performance of this study. The author also expresses gratitude to the participants for their collaboration.

Declaration of interest: No conflict of interest is declared by author.

Availability of data and materials: All data generated or analyzed during this study are included in this published article.

\section{REFERENCES}

1. Mills SEE, Nicolson KP, Smith BH. Chronic pain: a review of its epidemiology and associated factors in populationbased studies. Brit J Anaesthesia. 2019;123(2):e273-83. https://doi.org/10.1016/j.bja.2019.03.023 PMid:31079836 PMCid:PMC6676152

2. O'Reilly A. La dépression et l'anxiété dans la douleur chronique: une revue de travaux. J de Thérapie Comportementale et Cognitive. 2011;21(4):126-31. https://doi.org/10.1016/j.jtcc.2011.08.003

3. Fleishman TT, Dreiher J, Shvartzman P. Pain in maintenance hemodialysis patients: A multicenter study. J Pain Symptom Manag. 2018;56(2):178-84. https://doi.org/ 10.1016/j.jpainsymman.2018.05.008 PMid:29775691

4. Puljak L, Burilovic E, Brkovic T. Prevalence and severity of pain in adult end-stage renal disease patients on chronic intermittent hemodialysis: a systematic review. PPA. 2016;1131. https://doi.org/10.2147/PPA.S103927 PMid: 27382261 PMCid:PMC4922783

5. Sadigova E, Ozkurt S, Yalcin AU. Pain assessment in hemodialysis patients. Cureus. 2020;12(2). https://doi.org/ 10.7759/cureus.6903 PMid:32190460 PMCid:PMC7061817

6. Scholz J, Finnerup NB, Attal N, Aziz Q, Baron R, Bennett MI, et al. The IASP classification of chronic pain for ICD-11: chronic neuropathic pain. 2019;160(1):7. https://doi.org/10.1097/j.pain.0000000000001365 PMid:30586071 PMCid:PMC6310153

7. Nejmi M. Étude préliminaire de validation de la version arabe du questionnaire "Brief Pain Inventory" dans la douleur cancéreuse. 2006;10.

8. Terkawi A, Tsang S, AlKahtani G, Al-Mousa S, Al Musaed S, AlZoraigi $U$, et al. Development and validation of Arabic version of the hospital anxiety and depression scale. Saudi J Anaesth. 2017;11(5):11. https://doi.org/10.4103/ sja.SJA_43_17 PMid:28616000 PMCid:PMC5463562

9. Zigmond AS, Snaith RP. The hospital anxiety and depression scale. Acta Psychiatr Scand. 1983;67(6):361-70. https://doi.org/10.1111/j.1600-0447.1983.tb09716.x PMid:6880820

10. El Harraqui R, Abda N, Bentata Y, Haddiya I. Évaluation et analyse de la douleur en hémodialyse chronique. Néphrologie \& Thérapeutique. 2014;10(7):500-6. https://doi.org/10.1016/j.nephro.2014.06.005 PMid:25449761

11. Davison SN, Jhangri GS. The impact of chronic pain on depression, sleep, and the desire to withdraw from dialysis in hemodialysis patients. J Pain Symptom Manag. 2005;30(5):465-73. https://doi.org/10.1016/j.jpainsymman. 2005.05.013 PMid:16310620

12. Bouattar T, Skalli Z, Rhou H, Ezzaitouni F, Ouzeddoun N, Bayahia R, et al. Evaluation et analyse de la douleur chez les hémodialysés chroniques. Néphrologie \& Thérapeutique. 2014;5(7):637-41. https://doi.org/10.1016/ j.nephro.2009.06.004 PMid:19625232 
13. Noto-Kadou-Kaza B, Sabi KA, Tsevi CM, Belhadi N, Ait S, Amekoudi EYM, et al. Douleur Chronique chez l'Hémodialysé au Maroc. 2015;16:5. https://doi.org/10.1016/j.nephro.2015.07.184

14. Breivik H, Collett B, Ventafridda V, Cohen R, Gallacher D. Survey of chronic pain in Europe: Prevalence, impact on daily life, and treatment. Eur J Pain. 2006;10(4):287. https://doi.org/10.1016/j.ejpain.2005.06.009 PMid:16095934

15. Campbell CM, Edwards RR. Ethnic differences in pain and pain management. Pain Manag. 2012;2(3):219-30. https://doi.org/10.2217/pmt.12.7 PMid:23687518 PMCid: PMC3654683

16. James SL, Abate D, Abate KH, Abay SM, Abbafati C, Abbasi $\mathrm{N}$, et al. Global, regional, and national incidence, prevalence, and years lived with disability for 354 diseases and injuries for 195 countries and territories, 1990-2017: a systematic analysis for the Global Burden of Disease Study 2017. The Lancet. 2018;392(10159):1789-858.

17. Venkat A, Kaufmann KR, Venkat K. Care of the end-stage renal disease patient on dialysis in the ED. Amer J Emerg Med. 2006;24(7):847-58. https://doi.org/10.1016/ j.ajem.2006.05.011 PMid:17098110

18. Harraqui RE, Abda N, Bentata Y, Haddiya I. Evaluation et analyse de l'insomnie en hémodialyse chronique. Pan Afr Med J. 2014;19:221. https://doi.org/10.11604/pamj.2014. 19.221.4444 PMid:25838849 PMCid:PMC4374896

19. Ben Salah FZ, Dziri C, Mokni M. Douleurs ostéoarticulaires chez le dialysé chronique et médecine physique et de réadaptation. J Réadaptation Médicale: Pratique et Formation en Médecine Physique et de Réadaptation. 2012;32(1):19-26.

https://doi.org/10.1016/j.jrm.2011.11.003

20. Kliuk - Ben Bassat O, Brill S, Sharon H. Chronic pain is underestimated and undertreated in dialysis patients: A retrospective case study. Hemodialysis Int. 2019;23(4):E104-E105. https://doi.org/10.1111/hdi.12736 PMid:30735315

21. Nagar VR, Birthi P, Salles S, Sloan PA. Opioid use in chronic pain patients with chronic kidney disease: A systematic review. Pain Med. 2017;18(8):1416-49. https://doi.org/ 10.1093/pm/pnw238 PMid:28339928

22. Pham P-CT, Toscano E, Pham P-MT, Pham P-AT, Pham SV, Pham P-TT. Pain management in patients with chronic kidney disease. Clin Kidney J. 2009;2(2):111-8. https://doi.org/10.1093/ndtplus/sfp001 PMid:25949305 PMCid:PMC4421348

23. Chabroux-Seffert A, Magnin B, Barrier C, Afiani-Soubeyrand $A$, Azzouz L, Olivier A, et al. Douleur en hémodialyse: mise en place de techniques d'apaisement non médicamenteuses. Néphrologie \& Thérapeutique. 2014;10(5):324-5.

https://doi.org/10.1016/j.nephro.2014.07.090

24. Quintiliano A, Oehmen T, Kirsztajn GM, Pegado R. Transcranial direct current stimulation in management of pain, mood, functionality, and quality of life in patients undergoing hemodialysis: a study protocol for a doubleblind controlled randomized trial. Trials. 2019;20(1):805. https://doi.org/10.1186/s13063-019-3769-6 PMid:31888699 PMCid:PMC6937834

25. Journet J. La douleur en dialyse. Journal des Maladies Vasculaires. 2015;40(2):96. https://doi.org/10.1016/ j.jmv.2014.12.115
26. Veys B, Huygues T, Bera-Louville A, Garet N, Blond S. Évaluation de la douleur chronique au travers du handicap. Douleur analg. 2006;19(1-2):3-12. https://doi.org/10.1007/ s11724-006-0001-8

27. Gamondi C, Galli N, Schönholzer C, Marone C, Zwahlen H, Gabutti L, et al. Frequency and severity of pain and symptom distress among patients with chronic kidney disease receiving dialysis. Swiss Med Wkly. 2013;43:w1375. https://doi.org/10.4414/smw.2013.13750 PMid:23443906

28. Coluzzi F. Assessing and treating chronic pain in patients with end-stage renal disease. Drugs. 2018;78(14):1459-79. https://doi.org/10.1007/s40265-018-0980-9 PMid:30206801

29. Hsu H-J, Yen C-H, Hsu K-H, Wu I-W, Lee C-C, Hung M-J, et al. Factors associated with chronic musculoskeletal pain in patients with chronic kidney disease. BMC Nephrol. 2014;15(1):6. https://doi.org/10.1186/1471-2369-15-6 PMid:24400957 PMCid:PMC3890529

30. Von Korff M, Dworkin SF, Le Resche L, Kruger A. An epidemiologic comparison of pain complaints. Pain. 1988;32(2):173-83. https://doi.org/10.1016/03043959(88)90066-8

31. Sqalli-Houssaini T, Ramouz I, Fahi Z, Tahiri A, Sekkat FZ, Ouzeddoun N, et al. Troubles anxio-dépressifs et qualité de l'hémodialyse. Néphrologie \& Thérapeutique. 2005;1(1):31-7. https://doi.org/10.1016/j.nephro.2005.01. 007 PMid:16895665

32. Dogan E, Erkoc R, Eryonucu B, Sayarlioglu H, Agargun MYA. Relation between depression, some laboratory parameters, and quality of life in hemodialysis patients. Renal Failure. 2005;27(6):695-9. https://doi.org/10.1080/ 08860220500242728 PMid: 16350820

33. Zouari L, Elleuch M, Feki I, Allouch C, Yaïch S, Thabet JB, et al. La dépression chez les malades hémodialysés chroniques: A propos de 106 cas.:7.

34. Semaan V, Noureddine S, Farhood L. Prevalence of depression and anxiety in end-stage renal disease: A survey of patients undergoing hemodialysis. Appl Nurs Res. 2018;43:80-5. https://doi.org/10.1016/j.apnr.2018.07.009 PMid:30220369

35. Untas A, Chauveau P. Vers une meilleure évaluation des aspects psychosociaux en néphrologie. Néphrologie \& Thérapeutique. 2008;4(4):228-30. https://doi.org/10.1016/ j.nephro.2008.03.002 PMid:18455488

36. Williams LJ, Jacka FN, Pasco JA, Dodd S, Berk M. Depression and pain: an overview. Acta Neuropsych. 2006;18(2):79-87. https://doi.org/10.1111/j.16015215.2006.00130.x PMid:26989796

37. Wessely S, Hindmarch I. Taking the pain out of depression: dual action antidepressants in the relief of pain in depression, fibromyalgia and other chronic pain syndromes. Hum Psychopharmacol Clin Exp. 2004;19(S1):S1-2. https://doi.org/10.1002/hup.617 PMid: 15378671

38. Casten RJ, Parmelee PA, Kleban MH, Lawton PM, Katz IR. The relationships among anxiety, depression, and pain in a geriatric institutionalized sample. Pain. 1995;61(2):271-6. https://doi.org/10.1016/0304-3959(94)00185-H

39. Abramowitz JS, Olatunji BO, Deacon BJ. Health anxiety, hypochondriasis, and the anxiety disorders. Behavior Therapy. 2007;38(1):86-94. https://doi.org/10.1016/ j.beth.2006.05.001 PMid:17292697

40. COCHINAIRE J-P. présentée et soutenue publiquement dans le cadre du troisième cycle de médecine générale Par.:112. 
41. Richardson JC, Ong BN, Sim J. Experiencing chronic widespread pain in a family context: giving and receiving practical and emotional support: Chronic widespread pain in a family context. Sociol Health Ill. 2007;29(3):347-65. https://doi.org/10.1111/j.1467-9566.2007.00496.x

PMid:17470216

42. Davison SN. Pain in hemodialysis patients: prevalence, cause, severity, and management. Am J Kidney Dis. 2003;42(6):1239-47. https://doi.org/10.1053/j.ajkd.2003.08. 025 PMid:14655196
43. Binik YM, Baker AG, Kalogeropoulos D, Devins GM, Guttmann RD, Hollomby DJ, et al. Pain, control over treatment, and compliance in dialysis and transplant patients.:9. 